\title{
Air strategy and Application of PP bi Directional Air Cylinder Positioning System
}

\author{
Chaohui Gai \\ \{ gaichaohuiwh@163.com\} \\ Wuhan Vocational College of Software and Engineering Wuhan 430205, China
}

\begin{abstract}
The characteristics of the cylinder and the characteristics of the valve are the main factors that affect the pneumatic position control system. Firstly the dynamic and static characteristics of the cylinder has carried on the theoretical analysis, to construct the cylinder through the experimental dynamics, the proportional directional control valve flow characteristics of the approximate mathematical model, of gas moving conveyor position control system, a nonlinear mathematical model is established, and by means of computer simulation technology on the system characteristics were studied, discussed the influence of system parameters on the performance of the system, that system open-loop frequency response characteristics. On the basis of this, the control strategy of the pneumatic proportional directional control system is studied. Because of the serious nonlinear system, it is difficult to obtain good control effect by using classical control methods (such as PID control), which is not suitable for the occasion of control object parameter variation and nonlinear degree. A new control algorithm is proposed in this paper, that is, zero - pole assignment self-tuning adaptive control algorithm, combined with PID adjustment, combined with on-site integration. The results showed that compared with only using PID control, the proposed control strategy can significantly improve the system dynamic performance.
\end{abstract}

Keywords: Pneumatic servo positioning system; mathematical model; proportional valve; MCGS; conveyor

\section{Introduction}

Pneumatic technology is a new technology of a thriving, pneumatic components, pneumatic technology is one of the most important part of, with pneumatic components of drive and control system has been widely used in various sectors of the national economy of complete sets of equipment and automation production line. Pneumatic technology is a technology to realize the automation of production process by using the energy and signal transmission of compressed gases (such as compressed air or inert gas and hot gas) as the working media. It contains two aspects of pneumatic transmission and pneumatic control. Human beings are using air as the medium to transmit energy, which can be traced back thousands of years ago. But really on the nature and the basic principle of system research is started from the 1940s [1], formed to dynamics of pneumatic drive system and gas control system theory is the main content of the course - pneumatic system theory. At present, hydraulic and pneumatic is two are widely used in fluid power transmission and control, both of which have many similarities, there are also many different points, pneumatic technology really for the various fields all over the world accepted and widely used, is due to the 
rationalization of the production automation and operation sequence of the increasingly urgent need, but also because of the pneumatic technology has many advantages:

1) Pneumatic technology with air as the working medium, the air is desirable, and the viscosity is small, small flow resistance in the tube, easy to focus on gas supply and longdistance transmission. Therefore, as the working medium of the physical properties of compressed air, so that the pneumatic technology in a wide range of applications with environmental protection, safety, convenience and low cost advantages.

2) Pneumatic components are simple in structure and low in price, and can be used in the air to discharge into the atmosphere.

3) The pneumatic system is clean, even if there is a leak, it will not pollute the product and the environment as the hydraulic system.

4) Pure pneumatic control system is not subject to electromagnetic interference, there is no spark of danger, especially suitable for flammable or explosive potentially dangerous occasions.

5) Easy to carry out energy storage, can be used for emergency or system.

6) Air pressure transmission itself has overload protection performance. Pneumatic actuator can work under full load for a long time, it will automatically stop when overload.

7) Pneumatic components moving speed is high. General cylinder's movement speed is generally $0.05-1.5 \mathrm{~m} / \mathrm{s}$, some as high as $2-3 \mathrm{~m} / \mathrm{s}$, the high speed air cylinder can reach $15 \mathrm{~m} / \mathrm{s}$ above.

\section{Characteristics of Pneumatic Proportional Directional Control Valve}

In the late seventy "s, with the development of modern control theory and microelectronic technology, all kinds of low-cost, multi function and high performance integrated circuits have emerged in large numbers. Making use of an electronic device to complete the industrial robot, control of the flexible production line and other automation equipment, and gas hydraulic or pneumatic actuator gas fibrillation or other executing task, this trend for electric pneumatic control system has opened up a broad application prospect. The continuous improvement and development of micro electronic technology and computer control technology have laid a theoretical foundation for the development of electro pneumatic servo control technology. Especially in recent years, the United States, Germany and Japan and other industrial developed countries compete to invest considerable manpower and material resources in the research, and has made great progress. At the same time, all kinds of electrical servo control components has been constantly developing, maturing varieties and foreign each big famous gas dynamic component manufacturers [2], such as SMC, FESTO, herion, Martonair, Bosch and small gold well company have successfully developed a electric stretch ratio valve or electric - pneumatic servo valve, and has been put into the market. At home, Zhejiang University, there are four kinds of electro pneumatic proportional valve or electro pneumatic servo valve through the technical identification, the Beijing Institute of machine tool has also developed a electro pneumatic proportional valve. In addition, foreign countries have also appeared in the rodless cylinder, flexible piston rod cylinder, stepping pneumatic motor, pneumatic gripper and pneumatic gripper automatic switching unit model gas pneumatic components. 


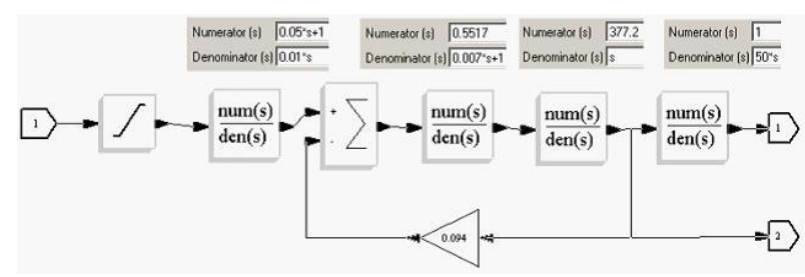

Fig. 1. Schematic diagram of system control system

\subsection{Characteristics of Pneumatic Servo Valve}

Valve is the control element of the cylinder, which is directly controlled by electric signal. It is also a signal converter. Five position three way valve can be used to control the movement of the cylinder, the valve core in the middle of the valve port closed, so to meet the cylinder at any location to stop. The valve must be continuously adjustable to allow for control of the flow and the movement of the control signal to be quickly converted to the required air pressure value. Pressure value must be able to be adjusted very precisely, based on this reason, the valve can be regarded as an independent closed-loop control link. In this part, the valve sliding displacement is measured and compared with the reference displacement. The two-way electromagnet has excellent dynamic characteristics, the dynamic response of the valve is very high frequency. Because the valve core is reset by the magnetic circuit of the bidirectional electromagnet, the electromagnet is not affected by the spring force load, so the power consumption is small, so that the whole electric control part can be integrated on the valve, and the amplifier is no longer needed in use. At the same time, because the valve core and the valve sleeve between the friction force and gas flow are in the control unit of the valve in the closed loop, so the control performance of the valve is almost no impact [3].
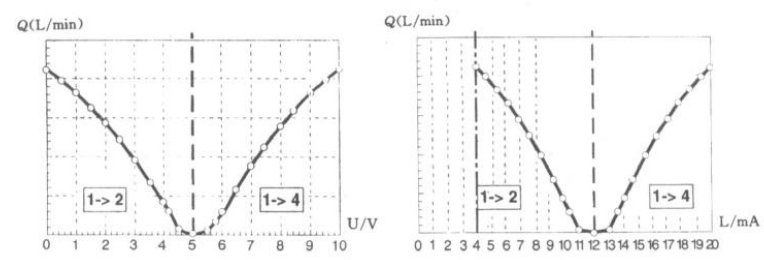

Fig. 2. Flow characteristics of MPYE type pneumatic servo valve

\subsection{The Basic Components of the Pneumatic Proportional Control System}

Compared with the hydraulic control system, the biggest difference lies in the compression of air and oil pressure and the difference of viscosity. The air has the characteristics of high compressibility and low viscosity, which is beneficial to form a soft driving mechanism and to realize high speed movement. In contrast, the compression of the assembly to bring the lag of the pressure response, viscous small means that the system damping is small or attenuation, easy to cause the vibration of the system response. Also due to the small damping, the gain coefficient of the system is not likely to be high, the stability of the system is prone to the influence of external disturbance and system parameters change, difficult to achieve high control precision, so in the past, people always think pneumatic control system can only be used at both ends of the cylinder stroke switch control, difficult to meet the position or force continuously adjustable high precision control requirements. 
Therefore, in the design of servo control system, in addition to some special occasions, the selection of pneumatic servo control system. But with the pneumatic proportional / servo control valve of the development and modern control theory into the model of the gas, the use of field bus technology, PLC controller and computer, making all kinds of new control mode, switch control PWM (pulse width modulation) control, PCM (pulse code modulation) can be application development, coupled with the gas dynamic system with light weight, cheap, anti electromagnetic interference and overload protection ability advantages, pneumatic proportional / servo control system is more and more by the designer's attention, its application field is constantly expanding. Especially to the controller SPC11 as the core of the gas dynamic servo positioning system, state feedback, dynamic compensation and other measures to improve the dynamic characteristics of the system, improve the control accuracy, positioning error is $0.2 \mathrm{~mm}$ [4], application in industrial production is getting more and more popular.

\section{Mathematical Model of Pneumatic Servo Positioning System}

Valve is an important control component in the pipeline transportation system. It is mainly used to control the pressure, flow and flow of the fluid. It is a kind of power device to control the output of the working mechanism by the input quantity. In a sense, pneumatic proportional control element of the advent and development is the organic combination of pneumatic technology and electronic technology and mechanical technology, realize gas electric integration and gas machine integration trend of development on behalf of. They through the electrical input signal of gas flow or pressure of continuous control, thus greatly simplifies the stepless or multilevel speed and output force gas actuated device of pneumatic and electric control circuit, and gas dynamic servo positioning feedback control system provides the essential elements.

In general, the digital simulation experiment includes three basic elements, namely the actual model, the mathematical model and the computer. The three elements are as follows: three basic activities, namely, model building, simulation experiment and result analysis. Will the actual model abstract for mathematical model, called a model. It also involves system identification techniques, collectively referred to as the modeling problem; mathematical model is converted to run on a computer simulation model, called the quadratic model, this involves the issue of simulation technology, collectively referred to as the simulation experiment. For a long time, the research emphasis in the field of simulation has been put on the establishment of the simulation model (that is, the two model problem), and thus a variety of simulation algorithms and tools are produced [5].

\section{Conclusion}

Characteristics of this paper for the gas dynamic position servo control system, the overall design, the installation of the gas conveyer position servo control system test platform, established the nonlinear mathematical model of the system, using computer simulation technology, the system characteristics and control strategy are studied, and through the experimental study to verify the feasibility of the proposed method. Pneumatic position servo system is a strong nonlinear system, the fundamental reason is with the compressibility of air, 
which makes saturation flow effect, the nonlinear friction factors exist in the system, to achieve high-precision positioning of the piston cylinder is very difficult. The in-depth study of the friction characteristics of the cylinder, the friction force is affecting the aerodynamic nonlinear system important reasons, due to the current from the theoretical analysis of characteristics of the friction of the cylinder is very difficult, so we use the integration of onsite, using the program segment called modified gain, so as to achieve better effect.

\section{Acknowledgements}

Scientific Research Program of Hubei Provincial Department of Education (No. B2015374).

\section{References}

[1] Qian Pengfei, Tao Guoliang, Liu Hao, Lu Bo, Zhong Wei. The characteristics of the cylinder crawl based on inlet throttle governing. Journal of Zhejiang University (Engineering Science Edition). No.07. (2012)

[2] Chen Jianfeng, Liu Hao, Tao Guoliang. Experimental. Journal of Lanzhou University of Technology on LuGre friction model Journal of. No. 03. (2010)

[3] Cao Jian, Zhu Xiaocong, Tao Guoliang. Identification of characteristic parameters and structural parameters in pneumatic servo control. Journal of Zhejiang University (Engineering Science Edition). No. 03. (2010)

[4] Wang Tao, Peng Guangzheng, Kagawa Leeharu. Measurement and synthesis method of small diameter pneumatic component flow characteristics. Journal of mechanical engineering. No. 06. (2012)

[5] Zhu Xiaocong, Tao Guoliang, Cao Jian. Trajectory tracking control of the position and orientation of pneumatic muscle parallel joint. Journal of mechanical engineering. No. 07. (2014) 